Sournal of Nonlinear Functional Analysis
Available online at http://jnfa.mathres.org

\title{
A MODIFIED HYBRID METHOD FOR SOLVING VARIATIONAL INEQUALITY PROBLEMS IN BANACH SPACES
}

\author{
YING LIU
}

College of Mathematics and Information Science, Hebei University, Baoding 071002, China

\begin{abstract}
In this paper, we propose a hybrid type algorithm without the extrapolation step for finding a solution of a variational inequality involving a monotone Lipschitz mapping in Banach spaces. Based on the generalized projection operator and the Lyapunov functional introduced by Alber, we obtain the strong convergence of the iterative sequence generated in the hybrid algorithm. Our results extend and improve the corresponding results in [Y.V. Malitsky, V.V. Semenov, A hybrid method without extrapolation step for solving variational inequality problems, J. Global Optim. 61 (2015), 193-202] and [K. Nakajo, Strong convergence for gradient projection method and relatively nonexpansive mappings in Banach spaces, Appl. Math. Comput. 271 (2015), 251-258].

Keywords. Hybrid method; Lyapunov functional; Generalized projection operator; Monotone mapping; Variational inequality.
\end{abstract}

2010 Mathematics Subject Classification. 47H05, 47N10, 90C33.

\section{Introduction}

Let $E$ be a real Banach space with norm $\|\cdot\|$ and let $E^{*}$ be the dual space of $E$. In this paper, we use $\langle x, f\rangle$ to denote the duality pairing of $E$ and $E^{*}$. Suppose that $C$ is a nonempty, closed and convex subset of $E$. In this paper, we study the problem of finding a point $u \in C$ such that

$$
\langle v-u, A u\rangle \geq 0, \quad \forall v \in C,
$$

E-mail address: 1y_cyh2013@163.com

Received December 6, 2016; Accepted May 13, 2017.

(C)2017 Journal of Nonlinear Functional Analysis 
where $A: C \rightarrow E^{*}$ is a given operator. This problem is said to be a variational inequality problem (VIP).

The variational inequality, which was first introduced by Lions and Stampacchia in 1967, has been intensively considered due to its various applications in operations research, economic equilibrium and engineering design. Various iterative methods for solving variational inequality (1.1) have been proposed and analyzed by many authors in Hilbert spaces or Banach spaces when $A$ has monotonicity and Lipschitz continuity or inverse-strong-monotonicity, see, for example, [2]-[15] and the reference therein.

In this paper, we focus on projection methods, which are the fundamental methods for solving VIPs with monotone and Lipschitz continuous mappings. Recently, Nakajo [2] introduced the following iterative algorithm.

\section{Algorithm 1.1.}

$$
\left\{\begin{array}{l}
x_{1}=x \in E, \text { arbitrarily chosen, } \\
y_{n}=\Pi_{C} J^{-1}\left(J x_{n}-\lambda_{n} A\left(x_{n}\right)\right), \\
z_{n}=T y_{n}, \\
C_{n}=\left\{u \in C: \phi\left(u, z_{n}\right) \leq \phi\left(u, x_{n}\right)-\phi\left(y_{n}, x_{n}\right)-2 \lambda_{n}\left\langle y_{n}-u, A x_{n}-A y_{n}\right\rangle\right\} \\
Q_{n}=\left\{u \in C:\left\langle x_{n}-u, J x-J x_{n}\right\rangle \geq 0\right\} \\
x_{n+1}=\Pi_{C_{n} \cap Q_{n}} x
\end{array}\right.
$$

where $E$ is a 2-uniformly convex and uniformly smooth Banach space, $T$ is a relatively nonexpansive mapping and $A$ is only supposed to be monotone and Lipschitz continuous. He proved sequence $\left\{x_{n}\right\}$ generated in (1.2) strongly converges to $\Pi_{D}(x)$, where $\Pi_{D}$ is the generalized projection onto $D$ and $D$ is the set of the common elements of the fixed points set of $T$ and the solution set of (1.1).

We observe that Algorithm 1.1 requires two generalized projections onto feasible sets $C$ and $C_{n} \cap Q_{n}$, and two times of computation of $A$ on every iteration. These might be costly when the feasible sets $C, C_{n} \cap Q_{n}$ and the operator $A$ have complex structures, as in large scale VIPs arising from optimal control; see [16] and the references therein. This might seriously affect the efficiency of the Algorithm 1.1. In this paper, we will construct a new iterative algorithm based on the idea in [17] as follows. 
In [17], Malitsky and Semenov introduced the following iterative algorithm.

\section{Algorithm 1.2.}

1. Choose $x_{0}, z_{0} \in C$ and two parameters $k>0$ and $\lambda>0$.

2. Given the current iterate $x_{n}$ and $z_{n}$, compute

$$
z_{n+1}=P_{C}\left(x_{n}-\lambda A\left(z_{n}\right)\right),
$$

where $P_{C}$ is the metric projection onto $C$. If $z_{n+1}=x_{n}=z_{n}$, then stop. Otherwise, construct sets $C_{n}$ and $Q_{n}$ as

$$
\left\{\begin{array}{l}
C_{0}=H, \\
C_{n}=\left\{w \in H:\left\|z_{n+1}-w\right\|^{2} \leq\left\|x_{n}-w\right\|^{2}+k\left\|x_{n}-x_{n-1}\right\|^{2}-\left(1-\frac{1}{k}-\lambda L\right)\left\|z_{n+1}-z_{n}\right\|^{2}\right. \\
\left.+\lambda L\left\|z_{n}-z_{n-1}\right\|^{2}\right\}, \quad n \geq 1, \\
Q_{0}=H, \\
Q_{n}=\left\{w \in H:\left\langle x_{n}-w, x_{0}-x_{n}\right\rangle \geq 0\right\}, \quad n \geq 1,
\end{array}\right.
$$

and calculate $x_{n+1}=P_{C_{n} \cap Q_{n}} x_{0}$, where $H$ is a Hilbert space.

3. Set $n \leftarrow n+1$ and return to step 2 .

They proved $\left\{x_{n}\right\}$ and $\left\{z_{n}\right\}$ generated in Algorithm 1.2 converge strongly to $P_{S}\left(x_{0}\right)$, where $S$ is the solution set of (1.1).

As remarked in [17], the sets $C_{n}$ and $Q_{n}$ in Algorithm 1.2 are halfspaces and hence it is much more simpler to calculate $P_{C_{n}} \cap Q_{n} x_{0}$ which can be found by Haugazeaus method ([18], Corollary 29.8) or any available method of convex quadratic programming ([19], Chapter 8) than projections onto the feasible sets. Thus, Algorithm 1.2 only needs to compute the projection onto the feasible set and the value of $A$ only one time. Hence, Algorithm 1.2 is easier to execute than Algorithm 1.1. Since the metric projection strictly depends on the inner product properties of Hilbert spaces, Algorithm 1.2 cannot be applied for variational inequality problem (1.1) in Banach spaces. However, many important problems related to practical problems are generally defined in Banach spaces. Therefore, it is meaningful to consider Problem (1.1) in Banach spaces.

Motivated and inspired by Algorithms 1.1 and 1.2, the purpose of this paper is to construct a new iterative algorithm for approximating a solution of problem (1.1) in Banach spaces. To 
be more clear, we will construct $C_{n}$ and $Q_{n}$ as half-spaces and only compute the value of $A$ one time on every iteration. The results presented in this paper mainly extend and improve the corresponding results in Nakajo [2], Malitsky and Semenov [17].

\section{Preliminaries}

Throughout this paper, let $\mathbb{N}$ be the set of all positive integers. We use $x_{n} \rightarrow x$ and $x_{n} \rightarrow$ $x$ to denote the strong convergence and weak convergence of the sequence $\left\{x_{n}\right\}$ in $E$ to $x$, respectively.

Let $U=\{x \in E:\|x\|=1\}$. A Banach space $E$ is said to be strictly convex if for any $x, y \in U$ and $x \neq y$ implies $\left\|\frac{x+y}{2}\right\|<1$. It is also said to be uniformly convex if for each $\varepsilon \in(0,2]$, there exists $\delta>0$ such that for any $x, y \in U,\|x-y\| \geq \varepsilon$ implies $\left\|\frac{x+y}{2}\right\| \leq 1-\delta$. It is known that a uniformly convex Banach space is reflexive and strictly convex. We define a function $\delta:[0,2] \rightarrow[0,1]$ called the modulus of convexity of $E$ as follows:

$$
\delta(\varepsilon)=\inf \left\{1-\left\|\frac{x+y}{2}\right\|: x, y \in U,\|x-y\| \geq \varepsilon\right\}
$$

Then $E$ is uniformly convex if and only if $\delta(\varepsilon)>0$ for all $\varepsilon \in(0,2]$. Let $p$ be a fixed real number with $p \geq 2$. A Banach space $E$ is said to be $p$-uniformly convex if there exists a constant $c>0$ such that $\delta(\varepsilon) \geq c \varepsilon^{p}$ for all $\varepsilon \in[0,2]$. It is obvious that a $p$-uniformly convex Banach space is uniformly convex; see [20] and [21] and the references therein. A Banach space $E$ is said to be smooth if the $\operatorname{limit}_{t \rightarrow 0} \frac{\|x+t y\|-\|x\|}{t}$ exists for all $x, y \in U$. It is also said to be uniformly smooth if the limit is attained uniformly for $x, y \in U$. It is well known that Hilbert and the Lebesgue $L^{q}(1<q \leq 2)$ spaces are 2-uniformly convex, uniformly smooth.

A Banach space $E$ is said to have the $K-K$ property if a sequence $\left\{x_{n}\right\}$ of $E$ satisfying that $x_{n} \rightarrow x \in E$ and $\left\|x_{n}\right\| \rightarrow\|x\|$, then $x_{n} \rightarrow x$. It is known that if $E$ is uniformly convex, then $E$ has the $K-K$ property.

Let $J: E \rightarrow 2^{E^{*}}$ be the normalized duality mapping defined by

$$
J(x):=\left\{v \in E^{*}:\langle x, v\rangle=\|v\|^{2}=\|x\|^{2}\right\}, \quad \forall x \in E .
$$

The following properties of the duality mapping $J$ can be found in [22]

(i) If $E$ is smooth, then $J$ is single-valued.

(ii) If $E$ is strictly convex, then $J$ is one-to-one and strictly monotone. 
(iii) If $E$ is uniformly smooth, then $J$ is uniformly norm-to-norm continuous on each bounded subset of $E$.

(iv) If $E$ is a smooth, strictly convex and reflexive Banach space, then $J$ is single-valued, one-to-one and onto and $J^{-1}$ is also single-valued, one-to-one, surjective, and it is the duality mapping from $E^{*}$ into $E$.

Let $E$ be a smooth Banach space. We consider the following Lyapunov functional introduced by Alber [23]

$$
\phi(x, y)=\|x\|^{2}-2\langle x, J y\rangle+\|y\|^{2}, \forall x, y \in E .
$$

Clearly, we have from the definition of $\phi$ that

(B1) $(\|x\|-\|y\|)^{2} \leq \phi(y, x) \leq(\|x\|+\|y\|)^{2}$,

(B2) $\phi(x, y)=\phi(x, z)+\phi(z, y)+2\langle x-z, J z-J y\rangle$,

(B3) $\phi(x, y)=\langle x, J x-J y\rangle+\langle y-x, J y\rangle \leq\|x\|\|J x-J y\|+\|y-x\|\|y\|$.

Let $E$ be a reflexive, strictly convex and smooth Banach space. $C$ denotes a nonempty, closed and convex subset of $E$. By Alber [23], for each $x \in E$, there exists a unique element $x_{0} \in C$ (denoted by $\Pi_{C}(x)$ ) such that

$$
\phi\left(x_{0}, x\right)=\min _{y \in C} \phi(y, x) .
$$

The mapping $\Pi_{C}: E \rightarrow C$, defined by $\Pi_{C}(x)=x_{0}$, is called the generalized projection operator from $E$ onto $C$. Moreover, $x_{0}$ is called the generalized projection of $x$. In a Hilbert space, $\Pi_{C}=P_{C}$ (the metric projection operator).

Lemma 2.1. [23] Let $C$ be a nonempty closed and convex subset of a smooth Banach space E and $x \in E$. Then, $x_{0}=\Pi_{C} x$ if and only if

$$
\left\langle x_{0}-y, J x-J x_{0}\right\rangle \geq 0, \quad \forall y \in C
$$

Lemma 2.2. [23] Let $E$ be a reflexive, strictly convex and smooth Banach space, let $C$ be a nonempty closed and convex subset of $E$ and let $x \in E$. Then

$$
\phi\left(y, \Pi_{C} x\right)+\phi\left(\Pi_{C} x, x\right) \leq \phi(y, x), \quad \forall y \in C .
$$

Lemma 2.3. [23] Let $C$ be a nonempty, closed and convex subset of a Banach space $E$ and $A$ a monotone, hemicontinuous operator of $C$ into $E^{*}$. Then

$$
V I(C, A)=\{u \in C:\langle v-u, A v\rangle \geq 0 \text { for all } v \in C\}
$$


It is obvious from Lemma 2.3 that set $\operatorname{VI}(C, A)$ is a closed and convex subset of $C$.

Lemma 2.4. [22] Let E be a 2-uniformly convex and smooth Banach space. Then

$$
\phi(x, y) \geq c_{1}\|x-y\|^{2}, \quad \forall x, y \in E,
$$

where $c_{1}>0$ is the 2-uniformly convexity constant of $E$.

Lemma 2.5. [17] Let $\left\{a_{n}\right\},\left\{b_{n}\right\},\left\{c_{n}\right\}$ be nonnegative real sequences, $\alpha, \beta \in R$ and for all $n \in N$ the following inequality holds

$$
a_{n} \leq b_{n}-\alpha c_{n+1}+\beta c_{n}
$$

If $\sum_{n=1}^{\infty} b_{n}<\infty$ and $\alpha>\beta \geq 0$, then $\lim _{n \rightarrow \infty} a_{n}=0$.

Lemma 2.6. [23] Let $E$ be a uniformly convex and smooth Banach space and let $\left\{y_{n}\right\},\left\{z_{n}\right\}$ be two sequences of $E$. If $\phi\left(y_{n}, z_{n}\right) \rightarrow 0$, and either $\left\{y_{n}\right\}$, or $\left\{z_{n}\right\}$ is bounded,then $y_{n}-z_{n} \rightarrow 0$.

\section{Main results}

Inspired by Malitsky and Semenov' results [17], we propose the Algorithm 3.1 to extend the Algorithm 1.2 from Hilbert spaces to Banach spaces and prove a strong convergence theorem, which is different from the scheme proposed by Nakajo [2].

In this section, we always assume the following conditions.

(C1) $E$ is a 2-uniformly convex and uniformly smooth Banach space with the 2-uniformly convexity constant $c_{1}$ and $C$ is a nonempty closed convex subset of $E$.

(C2) Mapping $A: C \rightarrow E^{*}$ is monotone and $L$-Lipschitz continuous.

(C3) The solution set of (1.1), denoted by $\operatorname{VI}(C, A)$, is nonempty.

\section{Algorithm 3.1.}

1. Choose $x_{0}, z_{0} \in C$ and two parameters $k>0$ and $\lambda>0$.

2. Given the current iterate $x_{n}$ and $z_{n}$, compute

$$
z_{n+1}=\Pi_{C} J^{-1}\left(J x_{n}-\lambda A\left(z_{n}\right)\right)
$$


where $\Pi_{C}$ is the generalized projection onto $C$. If $z_{n+1}=x_{n}=z_{n}$ then stop. Otherwise, construct sets $C_{n}$ and $Q_{n}$ as

$$
\left\{\begin{aligned}
C_{0}= & E \\
C_{n}= & \left\{w \in E: \phi\left(w, z_{n+1}\right) \leq \phi\left(w, x_{n}\right)+\left(\frac{k}{c_{1}}+\frac{1}{c_{1}^{2}}-1\right) \phi\left(x_{n-1}, x_{n}\right)\right. \\
& \left.\quad-\left(1-\frac{1}{c_{1} k}-\frac{\lambda L}{c_{1}}\right) \phi\left(z_{n+1}, z_{n}\right)+\frac{\lambda L}{c_{1}} \phi\left(z_{n}, z_{n-1}\right)\right\}, \quad n \geq 1, \\
Q_{0}= & E \\
Q_{n}= & \left\{w \in E:\left\langle x_{n}-w, J x_{0}-J x_{n}\right\rangle \geq 0\right\}, \quad n \geq 1,
\end{aligned}\right.
$$

and calculate $x_{n+1}=\Pi_{C_{n} \cap Q_{n}} x_{0}$.

3. Set $n \leftarrow n+1$ and return to step 2 .

Remark 3.2. Algorithm 3.1 improves Algorithm 1.1 in the following senses:

(1) Algorithm 3.1 improves $C_{n}$ and $Q_{n}$ from two general closed, convex sets to two halfspaces. Therefore, it is more easier to compute $\Pi_{C_{n} \cap Q_{n}}\left(x_{0}\right)$ than that in Algorithm 1.1 to obtain the next iterate.

(2) Algorithm 3.1 needs to compute the value of $A$ on each iteration only one time. Thus, Algorithm 3.1 reduces the number of computation required in Algorithm 1.1. Hence, Algorithm 3.1 improves Algorithm 1.1 constructed in [2] from a numerical point of view.

Lemma 3.3. If $z_{n+1}=x_{n}=z_{n}$ in Algorithm 3.1, then $x_{n} \in V I(C, A)$.

Proof. It follows from (3.1) and Lemma 2.1 that $\left\langle x_{n}-z, J x_{n}-\lambda A z_{n}-J x_{n}\right\rangle \geq 0, z \in C$. Since $\lambda>0$, we have $x_{n} \in V I(C, A)$. This completes the proof.

Theorem 3.4. Assume that (C1)-(C3) hold and let $\lambda \in\left(0, \frac{c_{1}}{2 L}\right), k>\frac{1}{c_{1}-2 \lambda L}$. Then $\left\{x_{n}\right\}$ and $\left\{z_{n}\right\}$ generated in Algorithm 3.1 converge strongly to $\Pi_{V I(C, A)}\left(x_{0}\right)$. 
Proof. It is evident that sets $C_{n}$ and $Q_{n}$ are closed and convex. Now, we show that $\operatorname{VI}(C, A) \subset C_{n}$ for all $n \in \mathbb{N}$. Let $u \in V I(C, A)$. By Lemma 2.2 and (B2), we have

$$
\begin{aligned}
& \phi\left(u, z_{n+1}\right) \leq \phi\left(u, J^{-1}\left(J x_{n}-\lambda A z_{n}\right)\right)-\phi\left(z_{n+1}, J^{-1}\left(J x_{n}-\lambda A z_{n}\right)\right) \\
& =\phi\left(u, x_{n}\right)+\phi\left(x_{n}, J^{-1}\left(J x_{n}-\lambda A z_{n}\right)\right)+2\left\langle u-x_{n}, J x_{n}-\left(J x_{n}-\lambda A z_{n}\right)\right\rangle \\
& -\phi\left(z_{n+1}, x_{n}\right)-\phi\left(x_{n}, J^{-1}\left(J x_{n}-\lambda A z_{n}\right)\right)-2\left\langle z_{n+1}-x_{n}, J x_{n}-\left(J x_{n}-\lambda A z_{n}\right)\right\rangle \\
& =\phi\left(u, x_{n}\right)-\phi\left(z_{n+1}, x_{n}\right)-2 \lambda\left\langle z_{n+1}-u, A z_{n}\right\rangle .
\end{aligned}
$$

Since $A$ is monotone and $u \in V I(C, A)$, we see that $\left\langle z_{n}-u, A z_{n}\right\rangle \geq 0$. It follows from (3.3) that

$$
\begin{aligned}
& \phi\left(u, z_{n+1}\right) \leq \phi\left(u, x_{n}\right)-\phi\left(z_{n+1}, x_{n}\right)-2 \lambda\left\langle z_{n+1}-z_{n}, A z_{n}\right\rangle \\
& =\phi\left(u, x_{n}\right)-\phi\left(z_{n+1}, x_{n-1}\right)-\phi\left(x_{n-1}, x_{n}\right)-2\left\langle z_{n+1}-x_{n-1}, J x_{n-1}-J x_{n}\right\rangle-2 \lambda\left\langle z_{n+1}-z_{n}, A z_{n}\right\rangle \\
& =\phi\left(u, x_{n}\right)-\phi\left(x_{n-1}, x_{n}\right)-2\left\langle z_{n+1}-x_{n-1}, J x_{n-1}-J x_{n}\right\rangle-\phi\left(z_{n}, x_{n-1}\right)-\phi\left(z_{n+1}, z_{n}\right) \\
& -2 \lambda\left\langle z_{n+1}-z_{n}, A z_{n}-A z_{n-1}\right\rangle+2\left\langle z_{n}-z_{n+1}, J z_{n}-J x_{n-1}+\lambda A z_{n-1} .\right.
\end{aligned}
$$

In view of $z_{n}=\Pi_{C} J^{-1}\left(J x_{n-1}-\lambda A z_{n-1}\right)$ and $z_{n+1} \in C$, we have

$$
\left\langle z_{n}-z_{n+1}, J z_{n}-J x_{n-1}+\lambda A z_{n-1}\right\rangle \leq 0 .
$$

Using Lemma 2.4, we obtain

$$
\begin{aligned}
& 2\left\langle z_{n+1}-x_{n-1}, J x_{n-1}-J x_{n}\right\rangle \\
& \leq 2\left\|z_{n+1}-x_{n-1}\right\|\left\|x_{n-1}-x_{n}\right\| \\
& \leq 2\left\|x_{n-1}-x_{n}\right\|\left(\left\|z_{n+1}-z_{n}\right\|+\left\|z_{n}-x_{n-1}\right\|\right) \\
& \leq 2 \frac{\sqrt{k} \sqrt{\phi\left(x_{n-1}, x_{n}\right)}}{\sqrt{c_{1}}} \frac{\sqrt{\phi\left(z_{n+1}, z_{n}\right)}}{\sqrt{k} \sqrt{c_{1}}}+2 \frac{\sqrt{\phi\left(x_{n-1}, x_{n}\right)}}{c_{1}} \sqrt{\phi\left(z_{n}, x_{n-1}\right)} \\
& \leq \frac{1}{c_{1}^{2}} \phi\left(x_{n-1}, x_{n}\right)+\phi\left(z_{n}, x_{n-1}\right)+\frac{k}{c_{1}} \phi\left(x_{n-1}, x_{n}\right)+\frac{1}{c_{1} k} \phi\left(z_{n+1}, z_{n}\right) .
\end{aligned}
$$

Since $A$ is Lipschitz continuous, we get

$$
\begin{aligned}
& 2 \lambda\left\langle z_{n+1}-z_{n}, A z_{n}-A z_{n-1}\right\rangle \leq 2 \lambda L\left\|z_{n+1}-z_{n}\right\|\left\|z_{n}-z_{n-1}\right\| \\
& \leq \lambda L\left(\left\|z_{n}-z_{n-1}\right\|^{2}+\left\|z_{n+1}-z_{n}\right\|^{2}\right) \leq \frac{\lambda L}{c_{1}}\left(\phi\left(z_{n+1}, z_{n}\right)+\phi\left(z_{n}, z_{n-1}\right)\right) .
\end{aligned}
$$


Combining inequalities (3.4)-(3.7), we see that

$$
\begin{aligned}
& \phi\left(u, z_{n+1}\right) \leq \phi\left(u, x_{n}\right)-\phi\left(x_{n-1}, x_{n}\right)-\phi\left(z_{n}, x_{n-1}\right)+\frac{1}{c_{1}^{2}} \phi\left(x_{n-1}, x_{n}\right)+\phi\left(z_{n}, x_{n-1}\right) \\
& +\frac{k}{c_{1}} \phi\left(x_{n-1}, x_{n}\right)+\frac{1}{c_{1} k} \phi\left(z_{n+1}, z_{n}\right)-\phi\left(z_{n+1}, z_{n}\right)+\frac{\lambda L}{c_{1}} \phi\left(z_{n}, z_{n-1}\right)+\frac{\lambda L}{c_{1}} \phi\left(z_{n+1}, z_{n}\right) \\
& =\phi\left(u, x_{n}\right)+\left(\frac{k}{c_{1}}+\frac{1}{c_{1}^{2}}-1\right) \phi\left(x_{n-1}, x_{n}\right)-\left(1-\frac{1}{c_{1} k}-\frac{\lambda L}{c_{1}}\right) \phi\left(z_{n+1}, z_{n}\right)+\frac{\lambda L}{c_{1}} \phi\left(z_{n}, z_{n-1}\right) .
\end{aligned}
$$

This shows that $\operatorname{VI}(C, A) \subset C_{n}$ for all $n \in \mathbb{N}$. Let us show by mathematical induction that $V I(C, A) \subset Q_{n}$ for all $n \in \mathbb{N}$. For $n=0$, we have $V I(C, A) \subset Q_{0}=E$. Suppose $V I(C, A) \subset Q_{n}$. It is sufficient to show that $\operatorname{VI}(C, A) \subset Q_{n+1}$. Since $x_{n+1}=\Pi_{C_{n} \cap Q_{n}} x_{0}$ and $V I(C, A) \subset C_{n} \cap Q_{n}$, we find that

$$
\left\langle x_{n+1}-z, J x_{0}-J x_{n+1}\right\rangle \geq 0, \quad \forall z \in V I(C, A) .
$$

This implies $z \in Q_{n+1}$. Thus, $\operatorname{VI}(C, A) \subset Q_{n+1}$ and hence $V I(C, A) \subset C_{n} \cap Q_{n}$ for all $n \in \mathbb{N}$. Hence, $\left\{x_{n}\right\}$ is well defined. Let $\bar{x}=\Pi_{V I(C, A)} x_{0}$. Since $x_{n+1}=\Pi_{C_{n} \cap Q_{n}} x_{0}$ and $\bar{x} \in V I(C, A) \subset$ $C_{n} \cap Q_{n}$, we have

$$
\phi\left(x_{n+1}, x_{0}\right) \leq \phi\left(\bar{x}, x_{0}\right)
$$

Therefore, $\left\{x_{n}\right\}$ is bounded. From $x_{n+1} \in C_{n} \cap Q_{n} \subset Q_{n}$ and $x_{n}=\Pi_{Q_{n}} x_{0}$, we obtain

$$
\phi\left(x_{n}, x_{0}\right) \leq \phi\left(x_{n+1}, x_{0}\right)
$$

Hence, $\lim _{n \rightarrow \infty} \phi\left(x_{n}, x_{0}\right)$ exists. In addition, since $x_{n}=\Pi_{Q_{n}} x_{0}$ and $x_{n+1} \in Q_{n}$, we from from Lemma 2.2 that

$$
\phi\left(x_{n+1}, x_{n}\right) \leq \phi\left(x_{n+1}, x_{0}\right)-\phi\left(x_{n}, x_{0}\right) .
$$

This implies $\sum_{n=1}^{\infty} \phi\left(x_{n+1}, x_{n}\right)<\infty$ is convergent. Since $x_{n+1} \in C_{n}$, we see that

$$
\begin{aligned}
\phi\left(x_{n+1}, z_{n+1}\right) \leq & \phi\left(x_{n+1}, x_{n}\right)+\left(\frac{k}{c_{1}}+\frac{1}{c_{1}^{2}}-1\right) \phi\left(x_{n-1}, x_{n}\right) \\
& -\left(1-\frac{1}{c_{1} k}-\frac{\lambda L}{c_{1}}\right) \phi\left(z_{n+1}, z_{n}\right)+\frac{\lambda L}{c_{1}} \phi\left(z_{n}, z_{n-1}\right) .
\end{aligned}
$$

Set $a_{n}=\phi\left(x_{n+1}, z_{n+1}\right), b_{n}=\phi\left(x_{n+1}, x_{n}\right)+\left(\frac{k}{c_{1}}+\frac{1}{c_{1}^{2}}-1\right) \phi\left(x_{n-1}, x_{n}\right), c_{n}=\phi\left(z_{n+1}, z_{n}\right), \alpha=(1-$ $\left.\frac{1}{c_{1} k}-\frac{\lambda L}{c_{1}}\right)$, and $\beta=\frac{\lambda L}{c_{1}}$. By Lemma 2.5, we have

$$
\lim _{n \rightarrow \infty} \phi\left(x_{n}, z_{n}\right)=0 .
$$

It follows from Lemma 2.6 that

$$
\lim _{n \rightarrow \infty}\left\|x_{n}-z_{n}\right\|=0
$$


Since $J$ is uniformly norm-to-norm continuous on bounded sets, we have

$$
\lim _{n \rightarrow \infty}\left\|J x_{n}-J z_{n}\right\|=\lim _{n \rightarrow \infty}\left\|J z_{n+1}-J z_{n}\right\|=0 .
$$

As $\left\{x_{n}\right\}$ is bounded, there exists a subsequence $\left\{x_{n_{i}}\right\}$ of $\left\{x_{n}\right\}$ such that $\left\{x_{n_{i}}\right\}$ converges weakly to some $x^{*} \in E$. Now, we show $x^{*} \in V I(C, A)$. Since $z_{n+1}=\Pi_{C} J^{-1}\left(J x_{n}-\lambda A z_{n}\right)$, it follows from Lemma 2.1 that

$$
\left\langle y-z_{n_{i}+1}, J z_{n_{i}+1}-J x_{n_{i}}+\lambda A z_{n_{i}}\right\rangle \geq 0, \forall y \in C
$$

This is equivalent to

$$
\begin{aligned}
0 & \leq\left\langle y-z_{n_{i}+1}, J z_{n_{i}+1}-J z_{n_{i}}+J z_{n_{i}}-J x_{n_{i}}\right\rangle \\
& +\lambda\left\langle y-z_{n_{i}}, A z_{n_{i}}\right\rangle+\lambda\left\langle z_{n_{i}}-z_{n_{i}+1}, A z_{n_{i}}\right\rangle \\
& \leq\left\langle y-z_{n_{i}+1}, J z_{n_{i}+1}-J z_{n_{i}}\right\rangle+\left\langle y-z_{n_{i}+1}, J z_{n_{i}}-J x_{n_{i}}\right\rangle \\
& +\lambda\left\langle y-z_{n_{i}}, A y\right\rangle+\lambda\left\langle z_{n_{i}}-z_{n_{i}+1}, A z_{n_{i}}\right\rangle, \quad \forall y \in C .
\end{aligned}
$$

In the last inequality, we used monotonicity of $A$. Taking the limit as $i \rightarrow \infty$ in (3.14) and using that $z_{n_{i}} \rightarrow x^{*}$, we obtain

$$
0 \leq\left\langle y-x^{*}, A y\right\rangle, \quad \forall y \in C,
$$

which implies by Lemma 2.3 that $x^{*} \in V I(C, A)$. Let us show $x_{n_{i}} \rightarrow x^{*}$. Since the norm of $E$ is weak lower semicontinuous, we get

$$
\begin{aligned}
& \phi\left(x^{*}, x_{0}\right)=\left\|x^{*}\right\|^{2}-2\left\langle x^{*}, J x_{0}\right\rangle+\left\|x_{0}\right\|^{2} \\
& \leq \liminf _{i \rightarrow \infty}\left(\left\|x_{n_{i}}\right\|^{2}-2\left\langle x_{n_{i}}, J x_{0}\right\rangle+\left\|x_{0}\right\|^{2}\right) \\
& =\liminf _{i \rightarrow \infty} \phi\left(x_{n_{i}}, x_{0}\right) \leq \limsup _{i \rightarrow \infty} \phi\left(x_{n_{i}}, x_{0}\right) \\
& \leq \phi\left(\Pi_{V I(C, A)} x_{0}, x_{0}\right) .
\end{aligned}
$$

The last inequality is due to (3.8). Since $x^{*} \in V I(C, A)$, we have $x^{*}=\Pi_{V I(C, A)} x_{0}$ and

$$
\lim _{i \rightarrow \infty} \phi\left(x_{n_{i}}, x_{0}\right)=\phi\left(x^{*}, x_{0}\right) .
$$

Again, we have $\lim _{i \rightarrow \infty}\left\|x_{n_{i}}\right\|=\left\|x^{*}\right\|$. Using the $K-K$ property of $E$, we obtain

$$
x_{n_{i}} \rightarrow x^{*}=\Pi_{V I(C, A)} x_{0} .
$$

Since $\left\{x_{n_{i}}\right\}$ is an arbitrary convergent subsequence of $\left\{x_{n}\right\}$, we find that $\left\{x_{n}\right\}$ converges strongly to $\Pi_{V I(C, A)} x_{0}$. It is clear that $z_{n} \rightarrow \Pi_{V I(C, A)} x_{0}$. This completes the proof. 
Remark 3.5. If $E$ is a Hilbert space, $c_{1}=1, \phi(x, y)=\|x-y\|^{2}, \Pi_{C}=P_{C}$, then Theorem 3.4 is reduced to Theorem 1 of Malitsky and Semenov [17].

\section{Conclusions}

The hybrid method without the extrapolation step was firstly introduced by Malitsky and Semenov [17] which provides a strong convergence theorem for variational inequalities of monotone and Lipschitz continuous operators in Hilbert spaces. In this paper, we extend Malitsky and Semenov's results from Hilbert spaces to Banach spaces. Our main results mainly improve the corresponding results in Nakajo [2] and Malitsky and Semenov [17].

\section{Acknowledgments}

This work was financially supported by the National Natural Science Foundation of China under Grant 11401157. The author is grateful to the reviewers for useful suggestions which improved the contents of this paper.

\section{REFERENCES}

[1] J.L. Lions, G. Stampacchia, Variational inequalities, Commun. Pure Appl. Math. 20 (1967), 493-517.

[2] K. Nakajo, Strong convergence for gradient projection method and relatively nonexpansive mappings in Banach spaces, Appl. Math. Comput. 271 (2015), 251-258.

[3] B.A. Bin Dehaish, A regularization projection algorithm for various problems with nonlinear mappings in Hilbert spaces, J. Inequal. Appl. 2015 (2015), Article ID 51.

[4] S. Y. Cho, B. A. Bin Dehaish, X. Qin, Weak convergence of a splitting algorithm in Hilbert spaces, J. Appl. Anal. Comput. 7 (2017), 427-438.

[5] J.M. Chen, L.J. Zhang, T.G. Fan, Viscosity approximation methods for nonexpansive mappings and monotone mappings, J. Math. Anal. Appl. 334 (2007), 1450-1461.

[6] Y. Liu, Strong convergence theorem for relatively nonexpansive mapping and inverse-strongly-monotone mapping in a Banach space, Appl. Math. Mech. Engl. Ed. 30 (2009), 925-932.

[7] X. Qin, S. Y. Cho, Convergence analysis of a monotone projection algorithm in reflexive banach spaces, Acta Math. Sci. Engl. Ed. 37 (2017), 488-502.

[8] Y. Censor, A. Gibali, S. Reich, The subgradient extragradient method for solving variational inequalities in Hilbert spaces, J. Optim. Theory Appl. 148 (2011), 318-335.

[9] N. Fang, Y. Gong, Viscosity iterative methods for split variational inclusion problems and fixed point problems of a nonexpansive mapping, Commun. Optim. Theory 2016 (2016), Article ID 11. 
[10] H. Zhou, P. Wang, A simpler explicit iterative algorithm for a class of variational inequalities in Hilbert spaces, J. Optim. Theory Appl. 161 (2014), 716-727.

[11] S.Y. Cho, Generalized mixed equilibrium and fixed point problems in a Banach space, J. Nonlinear Sci. Appl. 9 (2016), 1083-1092.

[12] X. Qin, J. C. Yao, Weak convergence of a Mann-like algorithm for nonexpansive and accretive operators, J. Inequal. Appl. 2016 (2016), Article ID 232.

[13] N. Buong, Strong convergence theorem of an iterative method for variational inequalities and fixed point problems in Hilbert spaces, Appl. Math. Comput. 217 (2010), 322-329.

[14] S. Lv, A new algorithm for solving nonlinear optimization problems, J. Nonlinear Funct. Anal. 2016 (2016), Article ID 2.

[15] X. Qin, S. Y. Cho, L. Wang, A regularization method for treating zero points of the sum of two monotone operators, Fixed Point Theory Appl. 2014 (2014), Article ID 75.

[16] J.L. Lions, Optimal Control of Systems Governed by Partial Differential Equations, Springer, Berlin (1971).

[17] Y.V. Malitsky, V.V. Semenov, A hybrid method without extrapolation step for solving variational inequality problems, J. Global Optim. 61 (2015), 193-202.

[18] H.H. Bauschke, P.L.Combettes, Convex Analysis and Monotone Operator Theory in Hilbert Spaces, Springer, New York, (2011).

[19] S. Boyd, L. Vandenberghe, Convex Optimization. Cambridge University Press, Cambridge, (2004).

[20] K. Ball, E.A. Carlen, E.H. Lieb, Sharp uniform convexity and smoothness inequalities for trace norms, Invent. Math. 115 (1994),463-482.

[21] Y. Takahashi, K. Hashimoto, M. Kato, On sharp uniform convexity, smoothness, and strong type, cotype inequalities, J. Nonlinear Convex Anal. 3 (2002),267-281.

[22] Y.I. Alber, S. Reich, An iterative method for solving a class of nonlinear operator equations in Banach spaces, Panamer. Math.J. 4 (1994), 39-54.

[23] Y.I. Alber, Metric and generalized projection operators in Banach spaces: Properties and applications, in: A.G. Kartsatos (Ed.), Theory and Applications of Nonlinear Operators of Accretive and Monotone Type, in: Lecture Notes Pure Appl. Math., vol. 178, Dekker, New York, 1996, pp. 15-50. 